\title{
Renal replacement therapy is
} independently associated with a lower risk of death in patients with severe acute kidney injury treated with targeted temperature management after out-ofhospital cardiac arrest

Yoon Hee Choi ${ }^{1}$, Dong Hoon Lee ${ }^{2}$, Je Hyeok Oh ${ }^{2^{*}}$ (D) Jung Hee Wee ${ }^{3}$, Tae Chang Jang ${ }^{4}$, Seung Pill Choi ${ }^{5}$, Kyu Nam Park ${ }^{6}$ and on behalf of the Korean Hypothermia Network Investigators

\section{Abstract}

Background: The effect of renal replacement therapy (RRT) on the outcomes of severe acute kidney injury (AKI) after out-of-hospital cardiac arrest (OHCA) is uncertain. This study aimed to evaluate the association of RRT with 6month mortality in patients with severe AKI treated with targeted temperature management (TTM) after OHCA.

Methods: This was a retrospective analysis of a prospectively collected multicentre observational cohort study that included adult OHCA patients treated with TTM across 22 hospitals in South Korea between October 2015 and December 2018. AKI was diagnosed using the Kidney Disease: Improving Global Outcomes criteria. The primary outcome was 6-month mortality and the secondary outcome was cerebral performance category (CPC) at 6 months. Multivariate Cox regression analysis was performed to define the role of RRT in stage 3 AKI.

* Correspondence: jehyeokoh@cau.ac.kr

${ }^{2}$ Department of Emergency Medicine, Chung-Ang University College of Medicine, 84, Heukseok-ro, Dongjak-gu, Seoul 06974, Republic of Korea

Full list of author information is available at the end of the article

C C The Author(s). 2020 Open Access This article is licensed under a Creative Commons Attribution 4.0 International License, which permits use, sharing, adaptation, distribution and reproduction in any medium or format, as long as you give appropriate credit to the original author(s) and the source, provide a link to the Creative Commons licence, and indicate if changes were made. The images or other third party material in this article are included in the article's Creative Commons licence, unless indicated otherwise in a credit line to the material. If material is not included in the article's Creative Commons licence and your intended use is not permitted by statutory regulation or exceeds the permitted use, you will need to obtain permission directly from the copyright holder. To view a copy of this licence, visit http://creativecommons.org/licenses/by/4.0/ The Creative Commons Public Domain Dedication waiver (http://creativecommons.org/publicdomain/zero/1.0/) applies to the data made available in this article, unless otherwise stated in a credit line to the data. 
(Continued from previous page)

Results: Among 10,426 patients with OHCA, 1373 were treated with TTM. After excluding those who died within $48 \mathrm{~h}$ of return of spontaneous circulation (ROSC) and those with pre-arrest chronic kidney disease, our study cohort comprised 1063 patients. AKI developed in 590 (55.5\%) patients and 223 (21.0\%) had stage 3 AKI. Among them, 115 (51.6\%) were treated with RRT. The most common treatment modality among RRT patients was continuous renal replacement therapy (111 [96.5\%]), followed by intermittent haemodialysis (4 [3.5\%]). The distributions of CPC (1-5) at 6 months for the non-RRT vs. the RRT group were 3/108 (2.8\%) vs. 12/115 (10.4\%) for CPC 1, 0/108 (0.0\%) vs. 1/ 115 (0.9\%) for CPC 2, 1/108 (0.9\%) vs. 3/115 (2.6\%) for CPC 3, 6/108 (5.6\%) vs. 6/115 (5.2\%) for CPC 4, and 98/108 (90.7\%) vs. 93/115 (80.9\%) for CPC 5, respectively $(P=0.01)$. The RRT group had significantly lower 6-month mortality than the non-RRT group (93/115 [81\%] vs. 98/108 [91\%], $P=0.04)$. Multivariate Cox regression analyses showed that RRT was independently associated with a lower risk of death in patients with stage 3 AKI (hazard ratio, 0.569 [95\% confidence interval, 0.377-0.857, $P=0.01]$ ).

Conclusion: Dialysis interventions were independently associated with a lower risk of death in patients with stage 3 AKI treated with TTM after OHCA.

Keywords: Renal replacement therapy, Acute kidney injury, Out-of-hospital cardiac arrest, Targeted temperature management, Therapeutic hypothermia

\section{Background}

Acute kidney injury (AKI) develops frequently after out-of-hospital cardiac arrest (OHCA) and is associated with long-term mortality and poor neurological outcomes [1-8]. Similar to critically ill patients, increased AKI severity is associated with increased mortality in OHCA patients $[2,4,9]$. In contrast, recovery from AKI was a potent predictor of survival and good neurological outcome at discharge [5]. Mortality rates of patients with stage 3 AKI after OHCA are reported to be over 70\% $[2,3]$. Although renal replacement therapy (RRT) is a treatment option for these patients, its impact on outcomes is uncertain. We hypothesised that applying RRT would be associated with better outcomes in patients who develop severe AKI after OHCA.

Two recent studies evaluated the effect of RRT on cardiac arrest outcomes [10, 11]. However, these studies did not show a risk reduction in patients receiving RRT. On the contrary, one of them reported a significant increase in mortality among patients receiving RRT [11]. The two studies did not consider AKI stage in their comparison of outcomes in patients with and without RRT. Because all patients who received RRT had stage 3 AKI, the risk of death in the RRT group was higher than that in the non-RRT group. Therefore, this study evaluated whether dialysis interventions had an impact on the outcomes of patients with stage $3 \mathrm{AKI}$ after OHCA who were treated with targeted temperature management (TTM).

\section{Material and methods}

\section{Study design and setting}

This was a retrospective analysis of the prospectively collected multicentre observational cohort study. Data were collected from the Korean Hypothermia Network (KORHN) prospective registry (KORHN-PRO) between
October 2015 and December 2018. The KORHN is a multicentre clinical research consortium for TTM in South Korea. In total, 22 academic hospitals participated in the KORHN-PRO.

\section{Study population}

All adult patients ( $\geq 18$ years) with OHCA, regardless of the cause of arrest, who were unconscious (Glasgow Coma Scale score $<8$ ) after the return of spontaneous circulation (ROSC) and treated with TTM, were enrolled in the registry. Patients with active intracranial bleeding, acute stroke, known limitations in therapy and do-notattempt resuscitation order, known pre-arrest cerebral performance category (CPC) 3 or 4, known disease making 6-month survival unlikely, and body temperature $<30^{\circ} \mathrm{C}$ on admission were excluded.

Enrolled patients received care for post-cardiac arrest syndrome (PCAS) according to standard operating procedures regarding $\mathrm{OHCA}$ at each hospital. The principal investigator of each participating hospital collected data from the hospital records of OHCA survivors treated with TTM. Using a telephone survey, the independent data input committee investigated the survival and neurological status 6 months after ROSC. If patients died before 6 months, the death date was recorded.

\section{Variables}

The primary outcome was 6-month mortality and the secondary outcome was CPC at 6 months. We examined the following variables: age, sex, body weight, medical history (hypertension, heart failure, diabetes mellitus), arrest location (home, workplace, street, public building, nursing home, unknown), witnessed arrest, bystander cardiopulmonary resuscitation (CPR), time from collapse to $\mathrm{CPR}$, time from $\mathrm{CPR}$ to ROSC, epinephrine dose, 
initial rhythm assessed by emergency medical service personnel in the field (ventricular fibrillation, pulseless ventricular tachycardia, pulseless electrical activity, asystole, unknown shockable, unknown non-shockable, unknown), arrest cause (medical or unknown, trauma, submersion, drug overdose, asphyxia, hanging), postROSC lactate, post-ROSC shock (cardiovascular sequential organ failure assessment score $\geq 1$ on day 1 ), targeted temperature of TTM $\left(33\right.$ or $\left.36^{\circ} \mathrm{C}\right)$, duration of TTM ( 24 or $48 \mathrm{~h}$ ), adverse events during the 7 days following ROSC (seizure, bleeding, sepsis, hypoglycaemia [blood glucose< $60 \mathrm{mg} / \mathrm{dl}$ ], sustained hyperglycaemia [blood glucose $>180$ $\mathrm{mg} / \mathrm{dl}$ for $>4 \mathrm{~h}$ ], tachycardia [ $>130 / \mathrm{min}$ ], and bradycardia $[<40 / \mathrm{min}]$ ), RRT modality (continuous renal replacement therapy [CRRT] or intermittent haemodialysis), RRT initiation time, reasons for initiating/terminating RRT, dialysis dependence at discharge, and serum creatinine $(\mathrm{SCr})$. The KORHN-PRO required input of data on $\mathrm{SCr}$ daily until 7 days after ROSC. If the SCr was checked twice or more in 1 day, the highest value was collected in the registry.

\section{Definition of AKI}

We defined AKI based on the diagnostic criteria stipulated in the Kidney Disease: Improving Global Outcomes (KDIGO) guidelines [12]. Because we did not have data on patients' prior kidney function, we used the "modification of diet in renal disease study" formula for glomerular filtration rate (GFR) to estimate baseline SCr using the lower end of the normal range of GFR [13]. After estimating baseline $\mathrm{SCr}$, we determined whether the patients had an AKI and subsequently classified them into AKI stages. A patient was defined as having AKI if one of the $\mathrm{SCr}$ measurements during the 7-day period was $\geq 150 \%$ of the baseline $\mathrm{SCr}$ or if the $\mathrm{SCr}$ increased $\geq 0.3 \mathrm{mg} / \mathrm{dl}$ within a 48-h interval during the 7-day period. Once AKI status was determined, AKI staging was performed. A 150199\%, 200-299\%, and $\geq 300 \%$ increase from baseline SCr in one of the $\mathrm{SCr}$ measurements during the 7-day period was defined as stage 1, 2, and 3 AKI, respectively. Regardless of fulfilling these criteria, patients were defined as developing stage 3 AKI when SCr increased $\geq 4.0 \mathrm{mg} / \mathrm{dl}$ or RRT was initiated. We could not use the criteria for hourly urine output because data on hourly urine output were not collected in the registry.

\section{Statistical methods}

Descriptive statistics are reported as median (interquartile range) or mean \pm standard deviation for continuous variables according to the normality of distribution. Data normality was analysed using Shapiro-Wilk test or Kolmogorov-Smirnov test. Categorical variables are reported as frequency (percentage). Demographics and clinical differences between groups were assessed using Pearson's chi-squared test, Fisher's exact test, independent sample $t$-test, or Mann-Whitney $U$ test, as appropriate. We performed multivariate Cox regression analysis to calculate the hazard ratio (HR) and 95\% confidence interval (CI) of RRT with respect to mortality and to find independent factors associated with risk of death. All variables were entered into a multivariate Cox regression analysis. Differences in Kaplan-Meier survival curves between RRT and non-RRT groups were compared using the log-rank test. A $P$ value $<01.05$ was considered statistically significant. Statistical analyses were performed using IBM SPSS version 25.0 (IBM Corp., Armonk, NY, USA).

\section{Results}

\section{Study population}

Out of 10,426 patients with OHCA that were screened during the study period, 1373 were treated with TTM at 22 academic hospitals throughout South Korea (Fig. 1). We excluded the patients who died within $48 \mathrm{~h}$ of ROSC to minimise competing risk, those with pre-arrest chronic kidney disease or end-stage renal disease, and those with incomplete data such as $\mathrm{SCr}$, survival, or neurological status at 6 months. In total, 1063 patients were enrolled in the study cohort. The baseline characteristics of stage 3 patients with AKI according to RRT status and outcomes are summarised in Tables 1 and 2.

\section{Descriptive data}

AKI was defined in 590/1063 (55.5\%) of OHCA patients treated with TTM. Most AKI developed within 3 days of ROSC (461/590 [78.1\%]). The frequency of AKI stage was $257 / 590$ [44.5\%], $110 / 590$ [18.6\%], and 223/590 [37.8\%] for stages 1,2 , and 3, respectively. RRT was applied in 115/223 (51.6\%) patients with stage 3 AKI. The most common modality among RRT patients was CRRT (111 [96.5\%]), and the remaining four patients $(3.5 \%)$ received intermittent haemodialysis. The average initiation time of RRT was 18 (9-46) h from the time that ROSC and RRT were initiated, which was within $48 \mathrm{~h}$ of ROSC in most cases $(89 / 115$ [77.4\%]). The time to achieve AKI stage 3 in patients with RRT was 1 (1-2) day, and the time to achieve AKI stage 3 in patients without RRT was 3 (2-4) days. Average SCr values from day 1 to 7 following ROSC is provided in the supplementary material (see Additional file 1, Table S1).

\section{Comparisons of 6-month mortality according to RRT status in different cohorts}

The overall 6-month mortality of all enrolled patients (entire cohort, $n=1063$ ) was $541 / 1063(50.9 \%)$. The 6-month mortality of the non-RRT group in the entire cohort was significantly lower than that of the RRT group (448/948 [47.3\%] vs. 93/115 [80.9\%], $P<0.001$ ) (Fig. 2). In case of patients with AKI (AKI cohort, $n=590$ ), 6-month mortality was $380 / 590(64.4 \%)$. The 6 -month mortality of the 


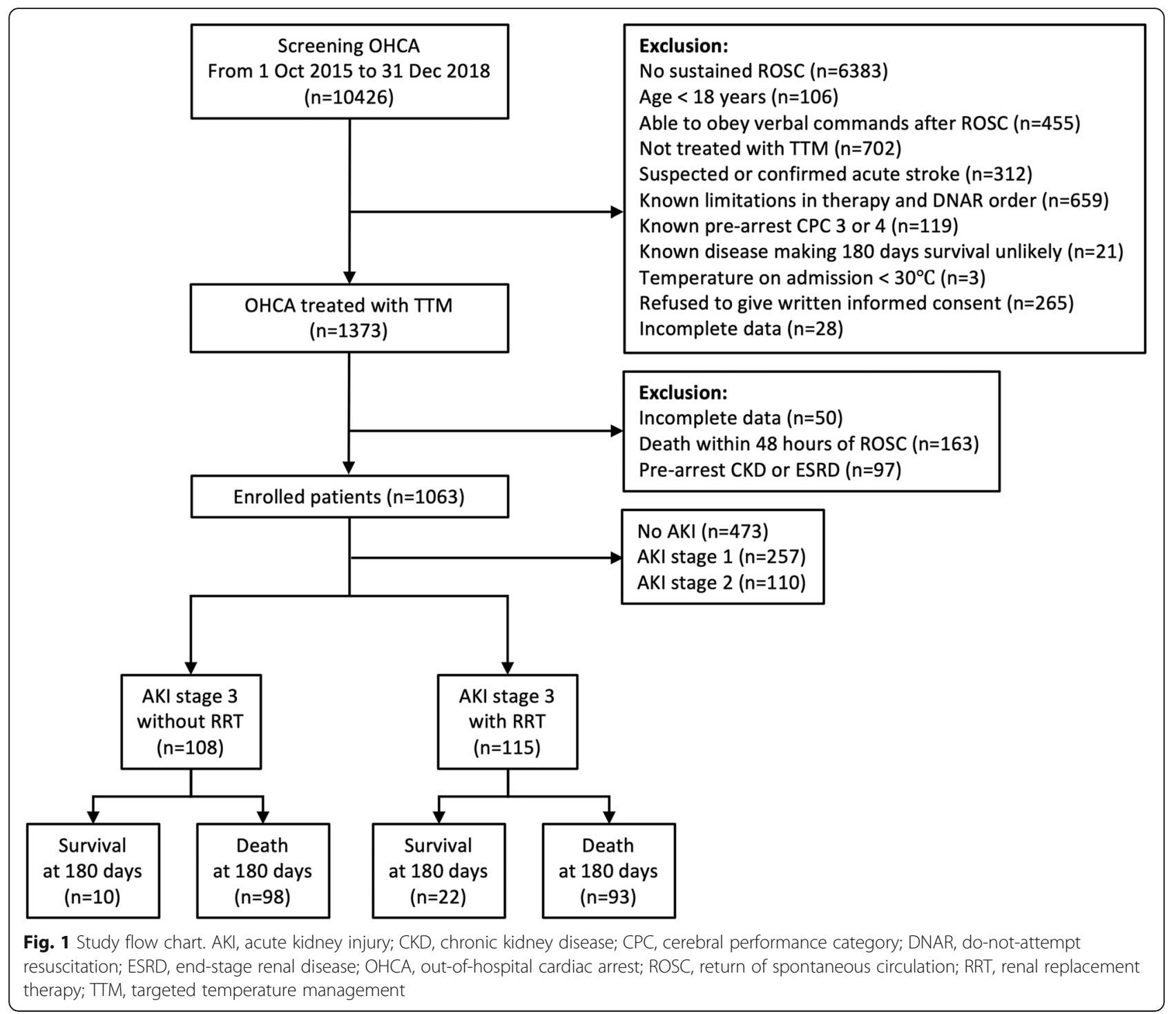

non-RRT group in AKI cohort also was significantly lower than that of the RRT group (287/475 [60.4\%] vs. 93/115 [80.9\%], $P<0.001)$. However, in patients with stage $3 \mathrm{AKI}$ (stage 3 AKI cohort, $n=223$ ), the 6-month mortality of the non-RRT group was significantly higher than that of the RRT group (98/108 [90.7\%] vs. 93/115 [80.9\%], $P=$ 0.04) (Table 2). The Kaplan-Meier survival curves differed significantly between the non-RRT and RRT groups in these cohorts $(P=0.003)$ (Fig. 3).

\section{Factors associated with 6-month mortality in patients} with stage $3 \mathrm{AKI}$

In the multivariate Cox regression analysis, four variables, namely, body weight, hypoglycaemia, re-arrest, and RRT, were independently associated with risk of death in patients with stage 3 AKI (Table 3). The HR of RRT was 0.569 (95\% CI, 0.377-0.857, $P=0.01)$.
Neurological outcomes according to the RRT status in patients with stage $3 \mathrm{AKI}$

The distributions of CPC (1-5) at 6 months for the nonRRT vs. RRT groups were $3 / 108$ (2.8\%) vs. $12 / 115$ (10.4\%) for CPC 1, 0/108 (0.0\%) vs. $1 / 115$ (0.9\%) for CPC 2, $1 / 108(0.9 \%)$ vs. 3/115 (2.6\%) for CPC 3, 6/108 (5.6\%) vs. 6/115 (5.2\%) for CPC 4, and 98/108 (90.7\%) vs. $93 / 115(80.9 \%)$ for $\mathrm{CPC} 5$, respectively $(P=0.01)$ (Table 2).

\section{Reasons for initiating and terminating RRT}

Oliguria or anuria was the leading cause of initiating RRT (62/115 [53.9\%]). Other causes followed acidosis (30/115 [26.1\%]), azotaemia (9/115 [7.8\%]), volume overload (8/115 [7.0\%]), and hyperkalaemia (6/115 [5.2\%]). Reasons for terminating RRT included death (67/115 [58.3\%]), recovery from AKI (20/115 [17.4\%]), withdrawal of RRT continuation by patient's legal surrogates 
Table 1 Patient characteristics according to the status of renal replacement therapy in patients who developed stage 3 acute kidney injury after out-of-hospital cardiac arrest

\begin{tabular}{|c|c|c|c|c|}
\hline & Total $(n=223)$ & Non-RRT group $(n=108)$ & RRT group $(n=115)$ & $P$ value \\
\hline \multicolumn{5}{|c|}{ Baseline characteristics } \\
\hline Age, years & $61(49-73)$ & $64(52-73)$ & $61(49-73)$ & 0.39 \\
\hline Male gender & $156(70.3)$ & $71(65.7)$ & $85(74.6)$ & 0.15 \\
\hline Body weight, kg & $69(60-75)$ & $65(59-73)$ & $69(60-75)$ & 0.16 \\
\hline \multicolumn{5}{|l|}{ Past medical history } \\
\hline Hypertension & $104(46.6)$ & $55(50.9)$ & $49(42.6)$ & 0.21 \\
\hline Heart failure & $10(4.5)$ & $4(3.7)$ & $6(5.2)$ & 0.75 \\
\hline Diabetes mellitus & $65(29.1)$ & $35(32.4)$ & $30(26.1)$ & 0.30 \\
\hline
\end{tabular}

\section{Cardiac arrest}

Arrest location

Home
Workplace
Street
Public building
Nursing home
Unknown

$126(56.5)$

67 (62.0)

0.09

$10(4.5)$

$4(3.7)$

59 (51.3)

47 (21.1)

19 (17.6)

$6(5.2)$

30 (13.5)

$16(14.8)$

$28(24.3)$

$5(2.2)$

$1(0.9)$

14 (12.2)

5 (2.2)

$1(0.9)$

Witnessed arrest

138 (62.7)

62 (58.5)

4 (3.5)

$4(3.5)$

Bystander CPR

$136(62.1)$

64 (59.8)

76 (66.7)

0.21

$1(0-7)$

$3(0-8)$

40 (26-53)

$40(29-50)$

$72(64.3)$

0.50

Time from collapse to CPR, min

$2(1-7)$

$3(1-5)$

$1(0-7)$

0.58

Epinephrine, $\mathrm{mg}$

Initial rhythm

vf

Pulseless VT

48 (21.5)

$12(11.1)$

$40(26-53)$

0.75

$2(1-7)$

0.83

PEA

$1(0.4)$

$1(0.9)$

0.04

Asystole

47 (21.1)

24 (22.2)

91 (40.8)

55 (50.9)

Unknown shockable

Unknown non-shockable

4 (1.8)

2 (1.9)

$1(0.4)$

Unknown

31 (13.9)

$0(0.0)$

$14(13.0)$

$<0.001$

Arrest cause

$\begin{array}{ll}\text { Medical or unknown } & 168(75.3) \\ \text { Trauma } & 5(2.2) \\ \text { Submersion } & 5(2.2) \\ \text { Drug overdose } & 3(1.3) \\ \text { Asphyxia } & 12(5.4) \\ \text { Hanging } & 30(13.5)\end{array}$

68 (63.0)

3 (2.8)

$4(3.7)$

36 (31.3)

$0(0.0)$

$23(20.0)$

36 (31.3)

$2(1.7)$

$1(0.9)$

17 (14.8)

$100(87.0)$

$2(1.7)$

$1(0.9)$

$1(0.9)$

$2(1.7)$

$10(9.3)$

$2(1.7)$

$22(20.4)$

$8(7.0)$

\section{Post-resuscitation}

$\begin{array}{ll}\text { Lactate after ROSC, mmol/L } & 12.2(8.9-14.8) \\ \text { Shock after ROSC } & 210(94.2) \\ \pi T \text { of } 36^{\circ} \mathrm{C} & 47(21.1) \\ \pi \mathrm{TM} \text { duration of } 48 \mathrm{~h} & 5(2.2)\end{array}$

Adverse events during 7 days since ROSC 
Table 1 Patient characteristics according to the status of renal replacement therapy in patients who developed stage 3 acute kidney injury after out-of-hospital cardiac arrest (Continued)

\begin{tabular}{|c|c|c|c|c|}
\hline & Total $(n=223)$ & Non-RRT group $(n=108)$ & RRT group $(n=115)$ & $P$ value \\
\hline Bleeding & $16(7.2)$ & $3(2.8)$ & $13(11.3)$ & 0.02 \\
\hline Sepsis & $54(24.2)$ & $24(22.2)$ & $30(26.1)$ & 0.50 \\
\hline Hypoglycaemia & $44(19.7)$ & $18(16.7)$ & $26(22.6)$ & 0.27 \\
\hline Sustained hyperglycaemia & $153(68.6)$ & $72(66.7)$ & $81(70.4)$ & 0.55 \\
\hline Tachycardia & $49(22.1)$ & $24(22.2)$ & $25(21.9)$ & 0.96 \\
\hline Bradycardia & $6(2.7)$ & $3(2.8)$ & $3(2.6)$ & 1.00 \\
\hline Re-arrest & 55 (24.8) & 22 (20.6) & 34 (28.7) & 0.16 \\
\hline
\end{tabular}

Values are expressed as number (\%) or median (interquartile range)

$P<0.05$ are presented in bold

$C P R$ cardiopulmonary resuscitation, $P E A$ pulseless electrical activity, $R O S C$ return of spontaneous circulation, $R R T$ renal replacement therapy, $T T$ target temperature, TTM targeted temperature management, $V f$ ventricular fibrillation, $V T$ ventricular tachycardia

$(18 / 115[15.7 \%])$, and discharge/transfer of the patient $(10 / 115[8.7 \%])$.

\section{Dialysis dependence in patients applying RRT}

Only 32 RRT patients survived to discharge (32/115 [27.8\%]). Among them, 8/32 (25\%) still needed RRT at discharge.

\section{Discussion}

Until recently, there was limited evidence on the use of RRT in OHCA patients. Although AKI frequently occurs after OHCA, the recent guidelines did not comment on the role of RRT during PCAS because of insufficient data $[1-8,14,15]$. In this multicentre cohort of severe AKI after OHCA treated with TTM in South Korea, we found that the 6-month mortality of the RRT group was significantly lower than that of the non-RRT group. Moreover, dialysis intervention was associated with lower 6-month mortality in the multivariate Cox regression analysis.

In previous studies, the mortality of the RRT group was not lower than that of the non-RRT group [3, 4, 10, 11]. Winther-Jensen et al. reported that the 30-day mortality

Table 2 Outcomes according to the status of renal replacement therapy in patients who developed stage 3 acute kidney injury after out-of-hospital cardiac arrest

\begin{tabular}{lllll}
\hline & $\begin{array}{l}\text { Total } \\
(n=223)\end{array}$ & $\begin{array}{l}\text { Non-RRT group } \\
(n=108)\end{array}$ & $\begin{array}{l}\text { RRT group } \\
(n=115)\end{array}$ & $P$ value \\
\hline 6-month mortality & $191(85.7)$ & $98(90.7)$ & $93(80.9)$ & $\mathbf{0 . 0 4}$ \\
CPC at 6 months & & & $12(10.4)$ & $\mathbf{0 . 0 1}$ \\
CPC 1 & $15(6.7)$ & $3(2.8)$ & $1(0.9)$ & \\
CPC 2 & $1(0.4)$ & $0(0.0)$ & $3(2.6)$ & \\
CPC 3 & $4(1.8)$ & $1(0.9)$ & $6(5.2)$ & \\
CPC 4 & $12(5.4)$ & $6(5.6)$ & $93(80.9)$ & \\
CPC 5 & $191(85.7)$ & $98(90.7)$ & &
\end{tabular}

Values are expressed as number (\%) or median (interquartile range) $P<0.05$ are presented in bold

$C P C$ cerebral performance category, $R R T$ renal replacement therapy of a RRT group was significantly higher than that of a non-RRT group in the cohort of OHCA patients [11]. However, Beitland et al. reported that the 6-month mortality of a RRT group was not different from that of a non-RRT group in the cohort of patients with AKI after OHCA [4], and Geri et al. reported that there were no differences between the RRT and non-RRT groups in a cohort of patients with stage 3 AKI [3]. Considering those results, we compared the mortality between the non-RRT and RRT groups in the different cohorts (all enrolled patients, patients with AKI, and stage 3 patients with AKI). Interestingly, although the 6-month mortality of the RRT group was significantly higher than that of the non-RRT group in the cohorts of all enrolled patients and patients with AKI, the result was reversed in the cohort of patients with stage 3 AKI (Fig. 2). This might be a promising result for nephrologists and intensive care practitioners and implies the need for RRT in patents with stage 3 AKI after OHCA treated with TTM.

However, many factors should be considered in evaluating the results of the current study. First, there were differences in the baseline characteristics of the study cohorts between the present study and previous ones (e.g., age, male sex, witnessed arrest, bystander CPR, time from CPR to ROSC, and shock after ROSC) [3, 4, 10, 11]. Second, there are insufficient data to determine absolute indications and the optimal timing for initiation of RRT. Moreover, some clinicians tend to delay RRT when they suspect that patients may recover on their own and because of well-known risks of RRT itself, including hypotension, arrhythmia, membrane bioincompatibility, vascular access, and anti-coagulant administration [16]. As a result, the initiation of RRT is extremely variable and based primarily on empiricism and local institutional practice and resources [17]. Therefore, it will be difficult to determine whether to provide RRT based only on whether the patient developed stage 3 AKI after OHCA. The AKI Network stated that the indications for RRT must be viewed within the 


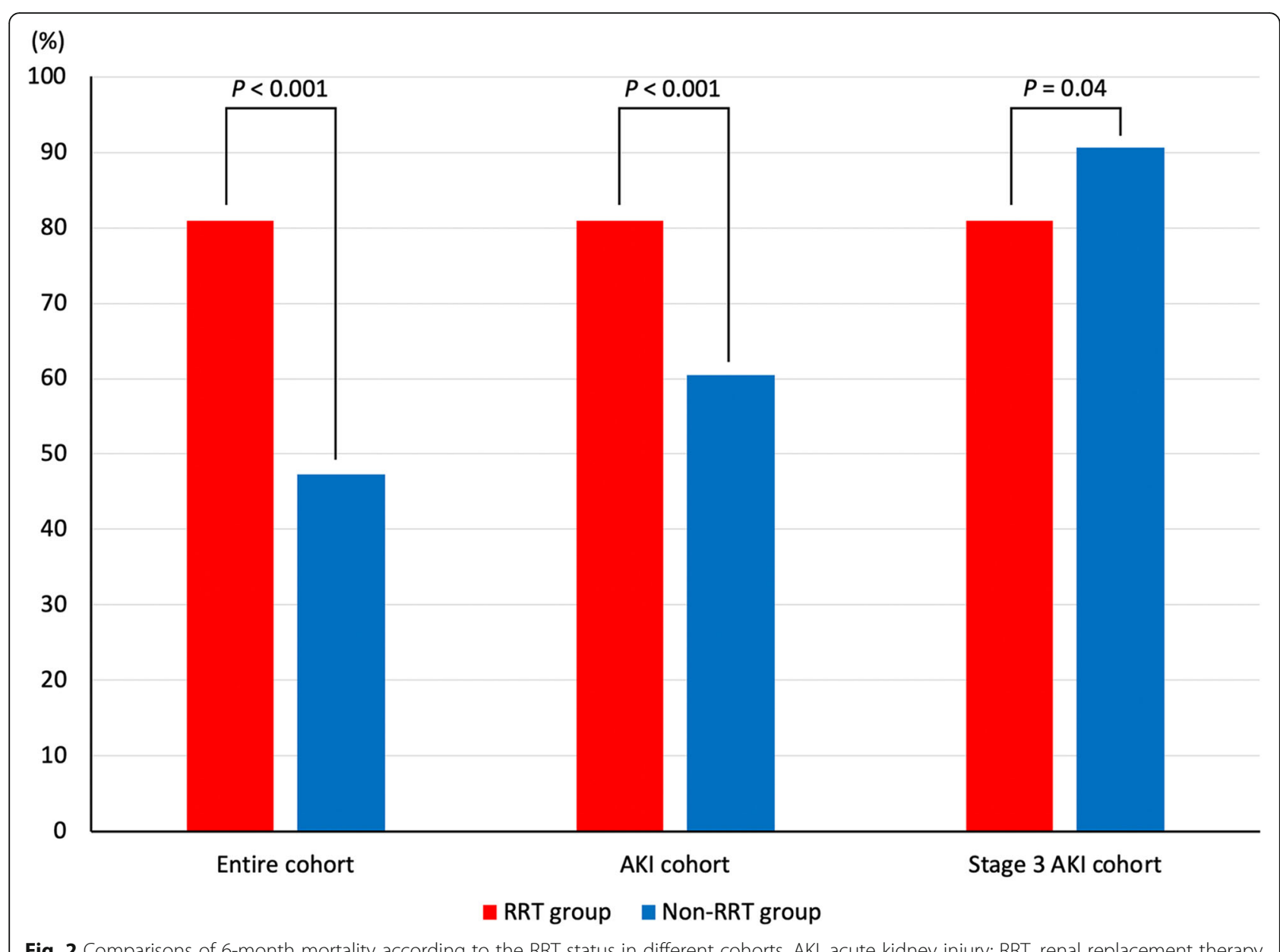

Fig. 2 Comparisons of 6-month mortality according to the RRT status in different cohorts. AKI, acute kidney injury; RRT, renal replacement therapy

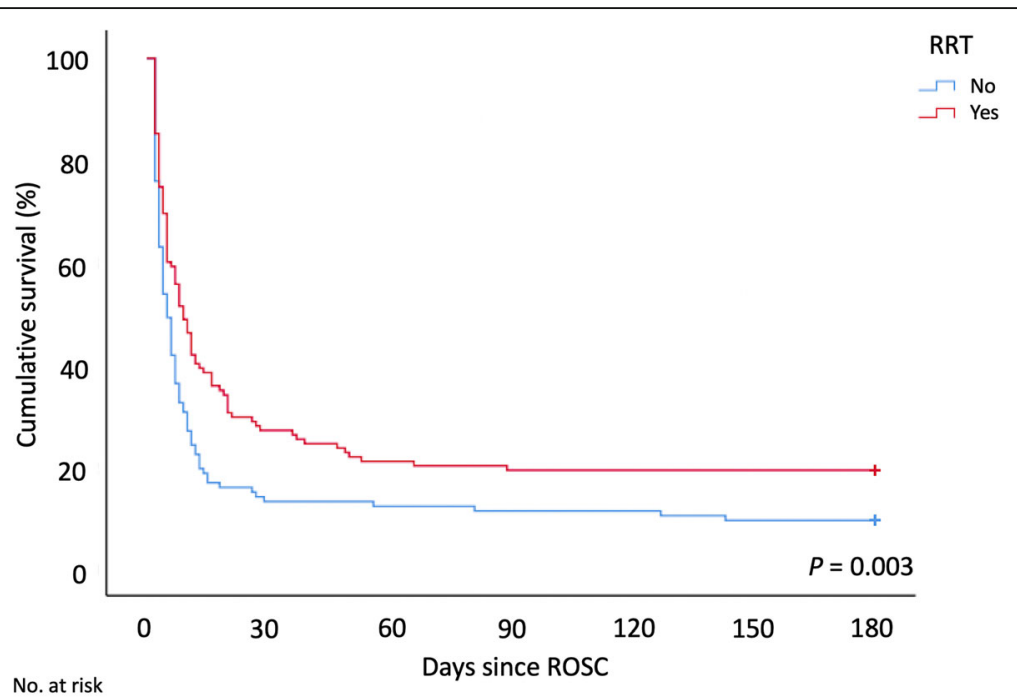

$\begin{array}{llllllll}\text { RRT } & 115 & 31 & 24 & 22 & 22 & 22 & 22 \\ \text { No RRT } & 108 & 14 & 13 & 12 & 12 & 10 & 10\end{array}$

Fig. 3 Cumulative survival up to 180 days since return of spontaneous circulation according to renal replacement therapy status. ROSC, return of spontaneous circulation; RRT, renal replacement therapy 
Table 3 Factors associated with 6-month mortality in patients who developed stage 3 acute kidney injury after an out-of-hospital cardiac arrest

\begin{tabular}{|c|c|c|c|c|c|c|}
\hline Variable & Coefficient & Standard error & Wald & $P$ value & Hazards ratio & $95 \% \mathrm{Cl}$ \\
\hline Age & 0.004 & 0.008 & 0.271 & 0.60 & 1.004 & $0.989-1.019$ \\
\hline Male gender & -0.033 & 0.221 & 0.022 & 0.88 & 0.967 & $0.628-1.491$ \\
\hline Body weight & 0.021 & 0.008 & 7.210 & 0.01 & 1.021 & $1.006-1.037$ \\
\hline Hypertension & -0.087 & 0.213 & 0.166 & 0.68 & 0.917 & $0.604-1.391$ \\
\hline Heart failure & -1.106 & 0.569 & 3.774 & 0.05 & 0.331 & $0.108-1.010$ \\
\hline Diabetes mellitus & -0.078 & 0.229 & 0.117 & 0.73 & 0.925 & $0.590-1.448$ \\
\hline Arrest location & & & & 0.05 & & \\
\hline Witnessed arrest & -0.299 & 0.233 & 1.654 & 0.20 & 0.741 & $0.470-1.170$ \\
\hline Bystander CPR & -0.219 & 0.268 & 0.669 & 0.41 & 0.803 & $0.475-1.359$ \\
\hline Time from collapse to CPR & 0.002 & 0.019 & 0.015 & 0.90 & 1.002 & $0.965-1.041$ \\
\hline Time from CPR to ROSC & -0.004 & 0.006 & 0.342 & 0.56 & 0.996 & $0.984-1.009$ \\
\hline Epinephrine, mg & 0.043 & 0.026 & 2.807 & 0.09 & 1.044 & $0.993-1.098$ \\
\hline Initial rhythm & & & & 0.42 & & \\
\hline Arrest cause & & & & 0.56 & & \\
\hline Lactate after ROSC & 0.034 & 0.022 & 2.535 & 0.11 & 1.035 & $0.992-1.080$ \\
\hline Shock after ROSC & 0.252 & 0.374 & 0.453 & 0.50 & 1.286 & $0.618-2.676$ \\
\hline TT of $36^{\circ} \mathrm{C}$ & -0.222 & 0.250 & 0.789 & 0.37 & 0.801 & $0.490-1.308$ \\
\hline TTM duration of $48 \mathrm{~h}$ & 0.461 & 0.714 & 0.417 & 0.52 & 1.586 & $0.392-6.421$ \\
\hline † Seizure & -0.472 & 0.251 & 3.526 & 0.06 & 0.624 & $0.381-1.021$ \\
\hline${ }^{\dagger}$ Bleeding & 0.333 & 0.364 & 0.838 & 0.36 & 1.395 & $0.684-2.845$ \\
\hline${ }^{\dagger}$ Sepsis & 0.264 & 0.228 & 1.351 & 0.25 & 1.303 & $0.834-2.035$ \\
\hline${ }^{\dagger}$ Hypoglycaemia & 0.462 & 0.230 & 4.038 & 0.04 & 1.587 & $1.011-2.489$ \\
\hline † Sustained hyperglycaemia & 0.257 & 0.206 & 1.561 & 0.21 & 1.294 & $0.864-1.937$ \\
\hline${ }^{\dagger}$ Tachycardia & -0.236 & 0.235 & 1.013 & 0.31 & 0.790 & $0.498-1.251$ \\
\hline${ }^{\dagger}$ Bradycardia & 0.069 & 0.684 & 0.010 & 0.92 & 1.071 & $0.281-4.089$ \\
\hline${ }^{\dagger}$ Re-arrest & 0.767 & 0.238 & 10.354 & 0.001 & 2.152 & $1.349-3.433$ \\
\hline RRT & -0.565 & 0.209 & 7.289 & 0.01 & 0.569 & $0.377-0.857$ \\
\hline
\end{tabular}

$P<0.05$ are presented in bold

$C P R$ cardiopulmonary resuscitation, ROSC return of spontaneous circulation, $R R T$ renal replacement therapy, $\Pi T$ target temperature, $T T M$ targeted

temperature management

${ }^{\dagger}$ Event recorded within 7 days since return of spontaneous circulation

context of the patient's entire clinical condition with most indications being relative; there are only a small number of absolute indications [17]. Third, the 6-month mortality of those in the RRT group with stage 3 AKI was extremely high in the present study. Moreover, a longer CPR time, lower ratio of shockable rhythm, and higher ratio of shock may underlie the high mortality in our cohort. In addition, a longer CPR time and lower ratio of shockable rhythm may underlie the higher ratio of shock in our cohort. Therefore, a large-scale multinational, multicentre study or randomised controlled trial will be needed to confirm the exact effect of RRT on the mortality after OHCA because of extremely high mortality in those with stage 3 AKI.
In case of critically ill patients, the overall in-hospital mortality in patients with AKI on dialysis consistently decreased, and the mortality of those with AKI on dialysis was relatively lower than that of the RRT group with OHCA; this is because outcomes associated with OHCA remain poor [18].

Contrary to the present study, some previous studies reported that the RRT was a risk factor for mortality even after correction for disease severity in case of critically ill patients, and the mortality of patients receiving RRT increased in the long-term follow-up over an 8year period $[19,20]$. Differences in disease severity, limits to defining disease severity accurately, and variability in selecting RRT could have contributed to the different results. Additional data from well-designed 
observational studies will be needed to clarify the longterm mortality of RRT group after OHCA.

Dialysis interventions were initiated within $48 \mathrm{~h}$ of ROSC in most cases, and the most common modality was CRRT. This may have been due to the high rate of shock after ROSC. These results are compatible with current standards because CRRT is recommended as the initial RRT modality [21]. A higher frequency of bleeding in the RRT group may have been caused by complications during the insertion of the dual-lumen haemodialysis catheter (Table 1). The ratio of dialysis dependence in our cohort was similar to that in a cohort of critically ill patients [20].

The distribution of CPC was significantly different according to the status of RRT (Table 2). Although only 32 patients with stage 3 AKI survived over 6 months, the high ratio of CPC 1 in the RRT group might suggest a beneficial effect of RRT. Further studies will be needed to verify the role of RRT on neurological outcomes after OHCA.

Although old age and initial non-shockable rhythm were risk factors for developing AKI after OHCA, age and differences in the initial rhythm between the nonRRT and RRT groups were not associated with 6-month mortality in the multivariate Cox regression analysis [1]. Instead, body weight, hypoglycaemia/re-arrest event within 7 days since ROSC, and RRT were associated with 6-month mortality. Interestingly, it was previously reported that overweight was associated with higher survival rate and a better neurological outcome after cardiac arrest [22]. Overweight per se might act protectively after cardiac arrest because of a better nutritive state compared with that of underweight. However, obesity is a well-known risk factor for AKI and mortality in critical illness [23]. As a result, an increase in body weight could act as a risk factor in our cohort of stage 3 AKI. Hypoglycaemia and re-arrest events were associated with poor outcome after cardiac arrest [24, 25]. Therefore, our results are compatible with previous studies.

\section{Limitations}

The present study has several limitations. First, because we defined AKI using only SCr criteria, it is possible that we underestimated AKI prevalence in our cohort. In the case of critically ill patients, the production of $\mathrm{SCr}$ decreases. Additionally, a decrease in $\mathrm{SCr}$ could be potentiated by therapeutic hypothermia [26]. Therefore, an exact judgement on AKI status is difficult in the case of individuals treated with therapeutic hypothermia. In addition, differences in urine output and fluid balance are likely to have influenced the decision to start RRT. However, data on urine output at RRT initiation and fluid balance could not be evaluated in our cohort. Second, the present study was a retrospective analysis of prospectively collected observational data that did not include information on standardised treatments or management protocols for RRT. Therefore, the risk of selection bias could have been introduced. Third, the initiation of RRT in our study was at the discretion of the physician responsible for the patient's care according to the KDIGO guideline (e.g., severe hyperkalaemia, severe acidosis, pulmonary oedema, and uremic complication). There were no consistent protocols dictating when to initiate RRT, which might have led to inconsistencies in practices among the principal investigators of each institution. A randomised controlled trial of RRT on patients with stage 3 AKI is needed to confirm the role of RRT. Fourth, there was a possibility of failure to conduct RRT even if urgent RRT was indicated because of the decision on withdrawal of life-sustaining therapy according to the neurological prognostication. However, in South Korea, the withdrawal of life-sustaining therapy depending on the neurological prognostication has not been used widely because of several reasons. For instance, the life-sustaining treatment decisions act was only applied recently in South Korea (since February 2018). In addition, legal surrogates, including family members, could not determine withdrawal of lifesustaining therapy, especially stopping the ventilator or removing the endotracheal tube owing to traditional sentiment. Therefore, we expect that decisions regarding withdrawal of life-sustaining therapy may not affect the initiation of RRT. However, there was a possibility that RRT may be stopped when a poor neurological outcome or death was expected through the treatment course, as the cost for RRT (especially continuous RRT) is quite expensive in South Korea (approximately $€ 400$ per day). Fifth, in the cohort of RRT $(n=115), 80$ patients were diagnosed as achieving stage 3 AKI by initiating RRT itself (Group A) and 35 patients were diagnosed as achieving stage 3 AKI based on SCr (Group B). Although the initiation of RRT was not determined by conditions other than $\mathrm{SCr}$ in most cases, daily SCr of Group B was significantly higher than that of Group A (see Additional file 1, Table S2). Therefore, it may be possible to compare patients reaching the AKI stage 3 by non-creatinine criteria with patients reaching the AKI stage 3 by creatinine criteria. The sixth limitation is that the results of the present study may have been affected by the characteristics of our study cohort. Therefore, the results might not be reproducible in another cohort with different characteristics. Fifth, although we excluded the patients who died within $48 \mathrm{~h}$ from the time of ROSC initiation to decrease problems related to competing risk, excluding these patients itself may have introduced bias.

\section{Conclusions}

Dialysis interventions were independently associated with a lower risk of death in patients with stage 3 AKI treated with TTM after OHCA. 


\section{Supplementary information}

Supplementary information accompanies this paper at https://doi.org/10. 1186/s13054-020-2822-x

Additional file 1: Supplementary material. Table S1. Average serum creatinine values from days 1 to 7 according to the cohort. Table S2. Comparisons of daily serum creatinine values between patients who were diagnosed with stage 3 acute kidney injury by initiating renal replacement therapy itself (Group A) and patients who were diagnosed with stage 3 acute kidney injury by serum creatinine value (Group B) in patients undergoing renal replacement therapy.

\section{Abbreviations}

AKI: Acute kidney disease; Cl: Confidence interval; CPC: Cerebral performance category; CPR: Cardiopulmonary resuscitation; CRRT: Continuous renal replacement therapy; GFR: Glomerular filtration rate; HR: Hazard ratio; KDIGO: Kidney Disease: Improving Global Outcomes; KORHN: Korean Hypothermia Network; KORHN-PRO: Korean Hypothermia Network prospective registry; OHCA: Out-of-hospital cardiac arrest; PCAS: Post-cardiac arrest syndrome; ROSC: Return of spontaneous circulation; RRT: Renal replacement therapy; SCr: Serum creatinine; TTM: Targeted temperature management

\section{Acknowledgements}

The following investigators participated in the Korean Hypothermia Network Network Chair: Seung Pill Choi (The Catholic University of Korea, Eunpyeong St. Mary's Hospital, emvic98@catholic.ac.kr); Principal investigators of each hospital: Kyu Nam Park (The Catholic University of Korea, Seoul St. Mary's Hospital), Minjung Kathy Chae (Ajou University Medical Center), Won Young Kim (Asan Medical Center), Byung Kook Lee (Chonnam National University Hospital), Dong Hoon Lee (Chung-Ang University Hospital), Tae Chang Jang (Daegu Catholic University Medical Center), Jae Hoon Lee (Dong-A University Hospital), Yoon Hee Choi (Ewha Womans University Mokdong Hospital), Je Sung You (Gangnam Severance Hospital), Young Hwan Lee (Hallym University Sacred Heart Hospital), In Soo Cho (Hanil General Hospital), Su Jin Kim (Korea University Anam Hospital), Jong-Seok Lee (Kyung Hee University Medical Center), Yong Hwan Kim (Samsung Changwon Hospital), Min Seob Sim (Samsung Medical Center), Jonghwan Shin (Seoul Metropolitan Government Seoul National University Boramae Medical Center), Yoo Seok Park (Severance Hospital), Hyung Jun Moon (Soonchunhyang University Hospital Cheonan), Won Jung Jeong (The Catholic University of Korea, St. Vincent's Hospital), Joo Suk Oh (The Catholic University of Korea, Uijeongbu St. Mary's Hospital), Seung Pill Choi (The Catholic University of Korea, Yeouido St. Mary's Hospital), Kyoung-Chul Cha (Wonju Severance Christian Hospital).

\section{Authors' contributions}

Wee JH contributed to the study conception and design. Choi YH, Lee DH, Oh JH, Wee JH, Jang TC, Choi SP, Park KN, and Korean Hypothermia Network Investigators contributed to data acquisition. Choi YH, Lee DH, and $\mathrm{Oh} \mathrm{JH}$ contributed to data analysis and interpretation. Oh JH contributed to statistical analysis and revisions. Oh JH contributed to acquisition of funding. Choi $\mathrm{YH}$ and $\mathrm{Oh} \mathrm{JH}$ contributed to the drafting of the manuscript and its critical revision for important intellectual content. All authors have read and approved the final version of the manuscript.

\section{Funding}

This work was supported by the National Research Foundation of Korea (NRF) grant funded by the Korea government (MSIT) (grant no. NRF2018R1C1B5082969).

\section{Availability of data and materials}

The datasets used and/or analysed during the current study are available from the corresponding author on reasonable request.

\section{Ethics approval and consent to participate}

The study design and plan, including the informed consent form, were approved by the institutional review board of each hospital. In accordance with national requirements and the principles of the Declaration of Helsinki, written informed consent was obtained from all patients' legal surrogates.

\section{Consent for publication}

Not applicable.

\section{Competing interests}

The authors declare that they have no competing interests.

\section{Author details}

${ }^{1}$ Department of Emergency Medicine, Ewha Womans University Medical Center and Ewha Womans University Mokdong Hospital, 1071, Anyangcheon-ro, Yangcheon-gu, Seoul 07985, Republic of Korea. ${ }^{2}$ Department of Emergency Medicine, Chung-Ang University College of Medicine, 84, Heukseok-ro, Dongjak-gu, Seoul 06974, Republic of Korea. ${ }^{3}$ Department of Emergency Medicine, Wonkwang University College of Medicine, Sanbon Hospital, 321, Snabon-ro, Gunpo-si, Gyeonggi-do 15865, Republic of Korea. ${ }^{4}$ Department of Emergency Medicine, Daegu Catholic University School of Medicine, 33, Duryugongwon-ro 17-gil, Nam-gu, Daegu 42472, Republic of Korea. ${ }^{5}$ Department of Emergency Medicine, Eunpyeong St. Mary's Hospital, The Catholic University of Korea College of Medicine, 1021, Tongil-ro, Eunpyeong-gu, Seoul 03312, Republic of Korea. ${ }^{6}$ Department of Emergency Medicine, Seoul St. Mary's Hospital, The Catholic University of Korea College of Medicine, 222, Banpo-daero, Seocho-gu, Seoul 06591, Republic of Korea.

Received: 11 December 2019 Accepted: 6 March 2020

Published online: 23 March 2020

\section{References}

1. Sandroni C, Dell'anna AM, Tujjar O, Geri G, Cariou A, Taccone FS. Acute kidney injury after cardiac arrest: a systematic review and meta-analysis of clinical studies. Minerva Anestesiol. 2016;82(9):989-99.

2. Oh JH, Lee DH, Cho IS, Youn CS, Lee BK, Wee JH, Cha KC, Chae MK, Shin J, Korean Hypothermia Network I. Association between acute kidney injury and neurological outcome or death at 6 months in out-of-hospital cardiac arrest: a prospective, multicenter, observational cohort study. J Crit Care. 2019:54:197-204.

3. Geri G, Guillemet L, Dumas F, Charpentier J, Antona M, Lemiale V, Bougouin W, Lamhaut L, Mira JP, Vinsonneau C, et al. Acute kidney injury after out-ofhospital cardiac arrest: risk factors and prognosis in a large cohort. Intensive Care Med. 2015;41(7):1273-80.

4. Beitland S, Nakstad ER, Staer-Jensen H, Draegni T, Andersen GO, Jacobsen D, Brunborg C, Waldum-Grevbo B, Sunde K. Impact of acute kidney injury on patient outcome in out-of-hospital cardiac arrest: a prospective observational study. Acta Anaesthesiol Scand. 2016;60(8):1170-81.

5. Park YS, Choi YH, Oh JH, Cho IS, Cha KC, Choi BS, You JS. Recovery from acute kidney injury as a potent predictor of survival and good neurological outcome at discharge after out-of-hospital cardiac arrest. Crit Care. 2019; 23(1):256.

6. Tujjar O, Mineo G, Dell'Anna A, Poyatos-Robles B, Donadello K, Scolletta S, Vincent $J \mathrm{~L}$, Taccone FS. Acute kidney injury after cardiac arrest. Crit Care. 2015;19:169.

7. Storm C, Krannich A, Schachtner T, Engels M, Schindler R, Kahl A, Otto NM Impact of acute kidney injury on neurological outcome and long-term survival after cardiac arrest - a 10year observational follow up. J Crit Care. 2018:47:254-9.

8. Hasslacher J, Barbieri F, Harler U, Ulmer H, Forni LG, Bellmann R, Joannidis M. Acute kidney injury and mild therapeutic hypothermia in patients after cardiopulmonary resuscitation - a post hoc analysis of a prospective observational trial. Crit Care. 2018;22(1):154

9. Hoste EA, Bagshaw SM, Bellomo R, Cely CM, Colman R, Cruz DN, Edipidis K, Forni LG, Gomersall CD, Govil D, et al. Epidemiology of acute kidney injury in critically ill patients: the multinational AKI-EPI study. Intensive Care Med. 2015;41(8):1411-23.

10. Ghoshal S, Yang V, Brodie D, Radhakrishnan J, Roh DJ, Park S, Claassen J, Agarwal S. In-hospital survival and neurological recovery among patients requiring renal replacement therapy in post-cardiac arrest period. Kidney Int Rep. 2019;4(5):674-8

11. Winther-Jensen M, Kjaergaard J, Lassen JF, Kober L, Torp-Pedersen C, Hansen SM, Lippert F, Kragholm K, Christensen EF, Hassager C. Use of renal replacement therapy after out-of-hospital cardiac arrest in Denmark 20052013. Scand Cardiovasc J. 2018;52(5):238-43. 
12. Section 2: AKI Definition. Kidney Int Suppl (2011) 2012, 2(1):19-36. https://doi.org/10.1038/kisup.2011.32. PMID: 25018918.

13. Bellomo R, Ronco C, Kellum JA, Mehta RL, Palevsky P. Acute dialysis quality initiative w: acute renal failure - definition, outcome measures, animal models, fluid therapy and information technology needs: the second international consensus conference of the acute dialysis quality initiative (ADQI) group. Crit Care. 2004;8(4):R204-12.

14. Wyllie J, Bruinenberg J, Roehr CC, Rudiger M, Trevisanuto D, Urlesberger B. European Resuscitation Council Guidelines for Resuscitation 2015: Section 7. Resuscitation and support of transition of babies at birth. Resuscitation. 2015;95:249-63.

15. Callaway CW, Donnino MW, Fink EL, Geocadin RG, Golan E, Kern KB, Leary M, Meurer WJ, Peberdy MA, Thompson TM, et al. Part 8: post-cardiac arrest care: 2015 American Heart Association guidelines update for cardiopulmonary resuscitation and emergency cardiovascular care. Circulation. 2015;132(18 Suppl 2):S465-82.

16. Section 5: Dialysis Interventions for Treatment of AKI. Kidney Int Suppl (2011) 2012, 2(1):89-115. https://doi.org/10.1038/kisup.2011.35. PMID: 25018921

17. Gibney N, Hoste E, Burdmann EA, Bunchman T, Kher V, Viswanathan R, Mehta RL, Ronco C. Timing of initiation and discontinuation of renal replacement therapy in AKl: unanswered key questions. Clin J Am Soc Nephrol. 2008;3(3):876-80.

18. Miyamoto $Y$, Iwagami $M$, Aso $S$, Yasunaga $H$, Matsui $H$, Fushimi $K$, Hamasaki $Y$, Nangaku M, Doi K. Temporal change in characteristics and outcomes of acute kidney injury on renal replacement therapy in intensive care units: analysis of a nationwide administrative database in Japan, 2007-2016. Crit Care. 2019;23(1):172

19. Elseviers MM, Lins RL, Van der Niepen P, Hoste E, Malbrain ML, Damas P. Devriendt $J$, investigators $S$ : renal replacement therapy is an independent risk factor for mortality in critically ill patients with acute kidney injury. Crit Care. 2010;14(6):R221.

20. De Corte W, Dhondt A, Vanholder R, De Waele J, Decruyenaere J, Sergoyne V, Vanhalst J, Claus S, Hoste EA. Long-term outcome in ICU patients with acute kidney injury treated with renal replacement therapy: a prospective cohort study. Crit Care. 2016;20(1):256.

21. Schneider AG, Bagshaw SM. Effects of renal replacement therapy on renal recovery after acute kidney injury. Nephron Clin Pract. 2014;127(1-4):35-41.

22. Ma Y, Huang L, Zhang L, Yu H, Liu B. Association between body mass index and clinical outcomes of patients after cardiac arrest and resuscitation: a meta-analysis. Am J Emerg Med. 2018;36(7):1270-9.

23. Danziger J, Chen KP, Lee J, Feng M, Mark RG, Celi LA, Mukamal KJ. Obesity, acute kidney injury, and mortality in critical illness. Crit Care Med. 2016;44(2): 328-34.

24. Bhardwaj A, Ikeda DJ, Grossestreuer AV, Sheak KR, Delfin G, Layden T, Abella BS, Leary M. Factors associated with re-arrest following initial resuscitation from cardiac arrest. Resuscitation. 2017;111:90-5.

25. Kim YM, Youn CS, Kim SH, Lee BK, Cho IS, Cho GC, Jeung KW, Oh SH, Choi $\mathrm{SP}$, Shin JH, et al. Adverse events associated with poor neurological outcome during targeted temperature management and advanced critical care after out-of-hospital cardiac arrest. Crit Care. 2015;19:283.

26. Zeiner A, Sunder-Plassmann G, Sterz F, Holzer M, Losert H, Laggner AN, Mullner M. The effect of mild therapeutic hypothermia on renal function after cardiopulmonary resuscitation in men. Resuscitation. 2004;60(3):253-61.

\section{Publisher's Note}

Springer Nature remains neutral with regard to jurisdictional claims in published maps and institutional affiliations.

Ready to submit your research? Choose BMC and benefit from:
- fast, convenient online submission
- thorough peer review by experienced researchers in your field
- rapid publication on acceptance
- support for research data, including large and complex data types
- gold Open Access which fosters wider collaboration and increased citations
- maximum visibility for your research: over 100M website views per year
At BMC, research is always in progress.
Learn more biomedcentral.com/submissions

